\title{
Three-Dimensional Navier-Stokes Calculations Using the Modified Space-Time CESE Method
}

\author{
Chau-Lyan Chang*
}

NASA Langley Research Center, Hampton, VA23681

\begin{abstract}
The space-time conservation element solution element (CESE) method is modified to address the robustness issues of high-aspect-ratio, viscous, near-wall meshes. In this new approach, the dependent variable gradients are evaluated using element edges and the corresponding neighboring solution elements while keeping the original flux integration procedure intact. As such, the excellent flux conservation property is retained and the new edge-based gradients evaluation significantly improves the robustness for high-aspect ratio meshes frequently encountered in three-dimensional, Navier-Stokes calculations. The order of accuracy of the proposed method is demonstrated for oblique acoustic wave propagation, shock-wave interaction, and hypersonic flows over a blunt body. The confirmed second-order convergence along with the enhanced robustness in handling hypersonic blunt body flow calculations makes the proposed approach a very competitive CFD framework for 3D Navier-Stokes simulations.
\end{abstract}

\section{Introduction}

I $\mathrm{n}$ recent years, the space-time conservation element solution element (CESE) method proposed by Chang [1] has attracted cross-discipline attention in computational aerosciences. Applications in different disciplines such as complex shock structure flow [2], aeroacoustic wave simulations [3], chemically reacting flow [4], magnetohodrodynamics [5], stress waves in solids[6], and the melting process [7-8] have emerged in the past decade. The method offers a genuine, multi-dimensional numerical framework for conservation laws that is second-order accurate in both space and time. Discretized equations are derived from reinforcing flux conservation locally as well as globally using the strong form of the governing equations. Numerical integration of the flux is carried out through a set of conservation elements (CE) that do not coincide with the solution elements (SE) where the dependent variables are assumed to vary linearly with respect to time and all spatial directions. This staggered arrangement of CE and SE completely eliminates the need to solve a Riemann problem along the element interface a practice that has been the central notion of many finite-volume [9-11], spectral difference [12], and discontinuous Galerkin (DG) methods [13-15]. The CESE method has been shown to provide remarkable numerical accuracy in the cross-discipline application mentioned above.

Despite decades of successfully applying modern upwind schemes in solving many supersonic problems with discontinuities using structured grids, it is becoming apparent that a straight forward extension of the numerical framework to calculations using an unstructured mesh may not work in many cases involving strong shocks [16-17]. In some applications, careful tuning of the flux limiters and entropy fixes may enhance the robustness of the schemes for unstructured meshes [18-19]. However, no universal fix is currently available. The problem rests on two main issues. The first is the lack of a multi-dimensional extension of the Riemann solver that is generally applicable to arbitrary triangular or tetrahedral meshes for the reconstruction of the interface flux. While dimensional splitting is often used to make the reconstruction locally one-dimensional for structured meshes, no proper counterpart is available for multi-dimensional unstructured meshes. The second issue is associated with the famous carbuncle phenomenon appearing in many hypersonic blunt body calculations [20-21]. Numerical instability near the bow shock of a blunt body causes the shock around the stagnation region to change shape, and the solution eventually diverges. Special treatment in the upwind formulation is required to maintain stability. In

\footnotetext{
${ }^{*}$ Research Scientist, Computational Aerosciences Branch, email: Chau-Lyan.Chang@nasa.gov, Senior member, AIAA
} 
some cases, the stabilized numerical solution still exhibits abnormal behavior inside the boundary layer near the stagnation region [16].

This paper focuses on applying the space-time CESE method for 3D Navier-Stokes calculations. In the past, the CESE method has been shown to provide accurate solutions of Navier-Stokes equations for several steady-state or time-accurate 2D problems [22]. Despite the remarkable success in solving the Euler equations, it has also been shown that when high aspect ratio viscous meshes are encountered, the CESE method becomes unstable owing to numerical problems with the dependent variable gradients in the elongated element [22]. The stiffness with high aspect ratio meshes is common to many numerical methods. However, the CESE method appears to be more sensitive because the dependent variable gradient plays an important role in the linearly varying solution element. The capability to handle a mesh with aspect ratio as high as $10^{5}-10^{6}$ is crucial for $3 \mathrm{D}$, Reynolds Averaged NavierStokes (RANS) or large eddy simulation (LES) calculations because, in general, a mesh with a $y^{+}$value of 1 or smaller is frequently encountered in these simulations.

The main objective of this paper is to investigate the validity of a modified CESE method proposed to deal with the high aspect ratio mesh problems in 3D, Navier-Stokes calculations. The modified scheme is aimed at improving the dependent variable gradient calculations that are problematic in high aspect ratio meshes. We discuss the modified scheme in detail in the next section. The order of accuracy of the proposed scheme is demonstrated for acoustic wave propagation, shock and wave interaction, and bow shocks over blunt bodies in the results section. Finally, some conclusions are drawn at the end of the paper.

\section{Numerical Method}

\section{The Original Space-time CESE method}

The numerical formulation of the space-time CESE method has been discussed in detail in the literature [1, 23,-24]. We briefly review the numerical formulation below before discussing the modified scheme. The three-dimensional compressible Navier-Stokes equations in vector form can be written as

$$
\frac{\partial Q}{\partial t}+\frac{\partial F}{\partial x}+\frac{\partial G}{\partial y}+\frac{\partial H}{\partial z}=K+\frac{\partial F_{v}}{\partial x}+\frac{\partial G_{v}}{\partial y}+\frac{\partial H_{v}}{\partial z}
$$

where the dependent variable vector is defined as $Q=(\rho, \rho u, \rho v, \rho w, e)$ and $x, y, z$, and $t$ represent spatial coordinates and time, respectively. Flow variables $\rho, u, v, w$, and $e$ represent density, three velocity components, and total energy $\left(e=p /(\gamma-1)+\rho\left(u^{2}+v^{2}+w^{2}\right) / 2\right)$, respectively. Definitions of the inviscid flux vectors $F, G$, $H$ and viscous flux vectors $F_{v}, G_{v}, H_{v}$ can be found in standard text books (e.g. Hirsch [25]) and will not be included here. The source vector $K$ contains all external forcing or other energy-related source terms. For axisymmetric flows, $H=H_{v}=0$ and $K$ contains extra terms associated with the cylindrical coordinate system. The Euler equations are recovered by neglecting all viscous and source terms on the right hand side of the equation. To close the system, the perfect gas relation, $p=\rho R T$ is used in conjunction with eq. (1).

The space-time CESE method is formulated in the strong form of the flux equations. The space-time flux $\vec{h}$ is defined as:

$$
\vec{h}=\left(F-F_{v}, G-G_{v}, H-H_{v}, Q\right)
$$

By using Gauss' divergence theorem in the space-time domain, eq. (1) is rewritten in the following integral form

$$
\oint_{S(V)} \vec{h} \cdot d \vec{s}+\int_{\mathrm{v}} K d V=0
$$


where the space-time flux vector is integrated over the surface $S$ of an arbitrary space-time volume $V$. The surface normal vector is defined by $\vec{s}=\vec{n} d A$, where $d A$ is the area increment on $S$, and $\vec{n}$ is the outward unit normal vector. Equation (3) is quite general, and in fact, any conservation law with or without a source vector can be cast into this form. Thus, the numerical algorithm devised for eq. (3) can be easily extended for other physical problems.

For an arbitrary 3D element with a solution point at $\left(x_{0}, y_{0}, z_{0}, t_{0}\right)$, the dependent variable vector is assumed to vary linearly within the element:

$$
Q(x, y, z, t)=Q_{0}+Q_{t}\left(t-t_{0}\right)+Q_{x}\left(x-x_{0}\right)+Q_{y}\left(y-y_{0}\right)+Q_{z}\left(z-z_{0}\right)
$$

where $Q_{0}$ is the solution vector at the solution point. The vectors $Q_{v}, Q_{x}, Q_{y}$, and $Q_{z}$ are analogous to the derivatives of the dependent variables. For a higher-order extension, quadratic or third order terms can be added. Theoretically, the solution point can be any point within the element. In conventional finite-volume based methods, the solution point is usually at the geometric center of the element; however, in the CESE method, the solution point is taken to be the geometric center of all surrounding integration volumes called conservation elements. As will be shown later, this definition leads to much simpler flux integration equations. The coefficients of the polynomial, $Q_{v}, Q_{v}, Q_{y}$, and $Q_{z}$, are part of the solutions and need to be determined within each element. Chang points out that the derivatives of dependent variables must be treated as unknowns in order to preserve the space-time inversion invariant property [26]. The use of eq. (4) results in a formally second-order accurate scheme.

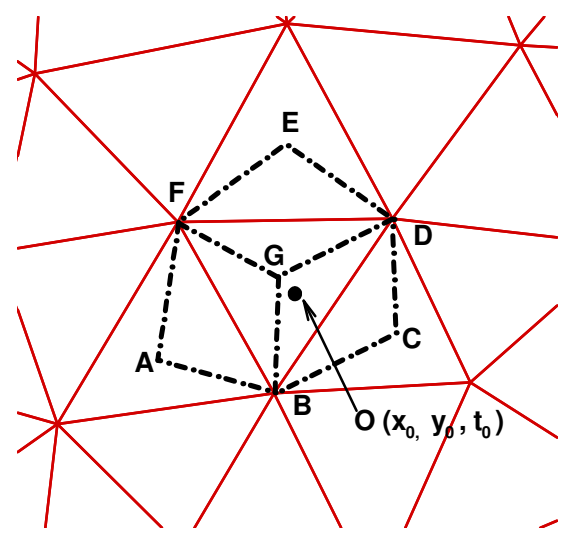

(a)

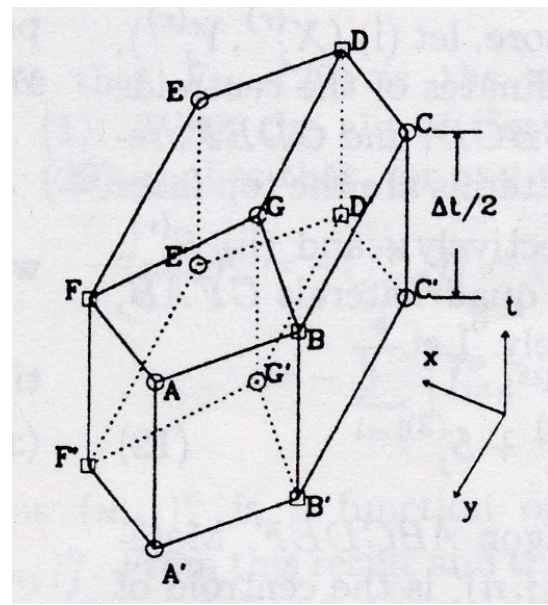

(b)

Figure 1. Conservation elements for a 2D triangular mesh: (a) top view (b) space time CE volume

Without loss of generality, we discuss the numerical formulation using a 2D triangular mesh depicted in Figure 1(a). Extension to tetrahedral and other types of elements is straightforward. Point $O$ with coordinates $\left(x_{0}, y_{0}, t_{0}\right)$ is the solution point of the element formed by $\triangle F B D$. For a cell-centered, finite-volume or discontinuous Galerkin method, numerical integration is done for $\triangle F B D$ directly. As a result, numerical fluxes must be evaluated at all the element interfaces $\overline{F B}, \overline{B D}$, and $\overline{D F}$. Unfortunately, numerical fluxes cannot be uniquely determined at these element interfaces due to its association with more than one element. For incompressible flows, a simple average of dependent variables from both sides of the interface is used to determine the $Q$ and flux vectors accordingly. For flows with discontinuities, an approximate Riemann solver is usually applied at the interface. While such Riemann solvers generally work well for 1D equations, no genuine multi-dimensional Riemann solver is available in the literature. A trivial extension using a 1D Riemann solver is usually imposed at the element interfaces. In contrast, the CESE method uses a different set of integration volumes called conservation elements (CE). Let points $G, A, C$, and $E$ denote the geometric centers of element $\triangle F B D$ and its three surrounding elements, respectively. Numerical results, as will be discussed later, indicate that using the in-centers (the intersection of three bi-sectors of three 
internal angles) [34] in lieu of geometric centers leads to better numerical accuracy. For the triangle $\triangle F B D$, three conservation elements formed by quadrilaterals $G B A F, G F E D$, and $G D C B$ are used for numerical integration of eq. (3). In the space-time domain, these quadrilaterals form three rectangular prisms with a height of $\Delta t / 2$ as shown in Fig. 1(b). Note that the bottom and top faces of these prisms are located at the old and new time level, respectively.

Two main steps are necessary to evaluate the unknowns of eq. (4) at the new time level. The first step involves evaluation of $Q_{0}$ at the new time level. Equation (4) is valid in the vicinity of the solution point $O$. The region of validity spans over three line segments: $\overline{G F}, \overline{G D}$, and $\overline{G B}$ at the new time level, and it extends $\Delta t / 2$ above and below point $O$. The flux integral (the first term in eq. (3)) is then carried out for all three CE prisms shown in Fig. 1(b). By summing up these three flux integrals, we obtain a discretized equation for the triangular element of interest. This discretized equation involves known values at the old-time level obtained from integrations over the outer-side ( $F A A^{\prime} F^{\prime}, A B B^{\prime} A^{\prime}$, etc) and bottom faces $\left(A^{\prime} B^{\prime} G^{\prime} F^{\prime}, G^{\prime} B^{\prime} C^{\prime} D^{\prime}\right.$, etc) of all three prisms. The flux integrals of the inner-side faces $\left(G F F^{\prime} G^{\prime}, G B B^{\prime} G^{\prime}\right.$, etc) cancel each other, and the only contribution at the new time level is associated with the top faces $(G B A F, G F E D$, and $G D C B)$. If the solution point $O$ is defined as the geometric center of the polygon $A B C D E F$, the discretized flux equation for all three prisms can be simplified to

$$
\left(A_{1}+A_{2}+A_{3}\right) Q_{0}=I_{1}+I_{2}+I_{3}+K_{v}
$$

where $A_{1}, A_{2}$, and $A_{3}$ are the surface areas of $G B A F, G F E D$, and $G D C B$, respectively. Discretized integrals $I_{1}, I_{2}$, and $I_{3}$ are flux integrals for outer-side and bottom faces for three surrounding elements. Source terms in eq. (3) can be integrated to form $K_{v}$. Semi-implicit treatment of this source term is usually done to improve solution accuracy and avoid stiffness issues in reacting flows [4]. Equation (5) is an algebraic equation and can be solved easily without any matrix conversion.

The second step involves evaluation of $Q_{t}, Q_{x}, Q_{y}$, and $Q_{z}$. In the non-dissipative a-scheme, these derivatives are computed by solving the individual flux equation associated with each $\mathrm{CE}$ prism in conjunction with the following equation:

$$
Q_{t}+A Q_{x}+B Q_{y}=K
$$

where $A$ and $B$ are the Jacobian matrices of the flux vectors $F$ and $G$, respectively. The above equation can be easily derived from eq. (1). Thus, there are a total of four equations for four unknowns. Unfortunately, the a-scheme is only neutrally stable for the nonlinear Euler and Navier-Stokes equations and cannot be used in general. Nevertheless, the existence of such a dissipation-free scheme serves as a baseline to gauge how much numerical dissipation is being added in the c-scheme discussed next. To add dissipation for any nonlinear conservation law, derivatives are evaluated using finite-differences. For the triangular element of interest, let $O_{1}\left(x_{1}, y_{1}\right), O_{2}\left(x_{2}, y_{2}\right)$ and $O_{3}\left(x_{3}, y_{3}\right)$ denote solution points of all three surrounding elements (see Fig. 2). On a two-dimensional plane, derivatives in $\mathrm{x}$ and $\mathrm{y}$ can be uniquely determined if any dependent variable is known at three distinct points that are not co-linear. From step one, dependent variable $Q_{0}$ is already known. Let $\widetilde{Q}_{0}^{i}$ ( $i=1$, 3 ) denote dependent variables at neighboring solution points evaluated by using the corresponding solution elements at the old time level

$$
\tilde{Q}_{0}^{i}=Q_{0}^{i}+Q_{t}^{i} \Delta t / 2
$$

where $Q_{0}^{i}$ and $Q_{t}^{i}$ are known solutions at the old time level. Three sets of derivatives can be obtained

$$
\begin{aligned}
& \left(Q_{x}^{1}, Q_{y}^{1}\right)=f_{d}\left(Q_{0}, \tilde{Q}_{0}^{1}, \tilde{Q}_{0}^{2}, O, O_{1}, O_{2}\right) \\
& \left(Q_{x}^{2}, Q_{y}^{2}\right)=f_{d}\left(Q_{0}, \widetilde{Q}_{0}^{2}, \tilde{Q}_{0}^{3}, O, O_{2}, O_{3}\right)
\end{aligned}
$$




$$
\left(Q_{x}^{3}, Q_{y}^{3}\right)=f_{d}\left(Q_{0}, \tilde{Q}_{0}^{3}, \tilde{Q}_{0}^{1}, O, O_{3}, O_{1}\right)
$$

The function $f_{d}$ denotes derivative evaluation using the three dependent variables and three solution points given. In the c-scheme, the derivatives at the new time level is then determined by applying a weighted average over the above three sets of derivatives:

$$
\begin{aligned}
& Q_{x}=W\left(Q_{x}^{1}, Q_{x}^{2}, Q_{x}^{3}\right) \\
& Q_{y}=W\left(Q_{y}^{1}, Q_{y}^{2}, Q_{y}^{3}\right)
\end{aligned}
$$

Details of the weighted averaging procedure can be found in ref.[1,23]. This weighting function returns the equivalent of a simple average for smooth flows and a biased value toward smaller derivatives when large gradients are encountered. It is analogous to the flux limiters used in conventional upwind schemes to contain large gradient or flow discontinuities.

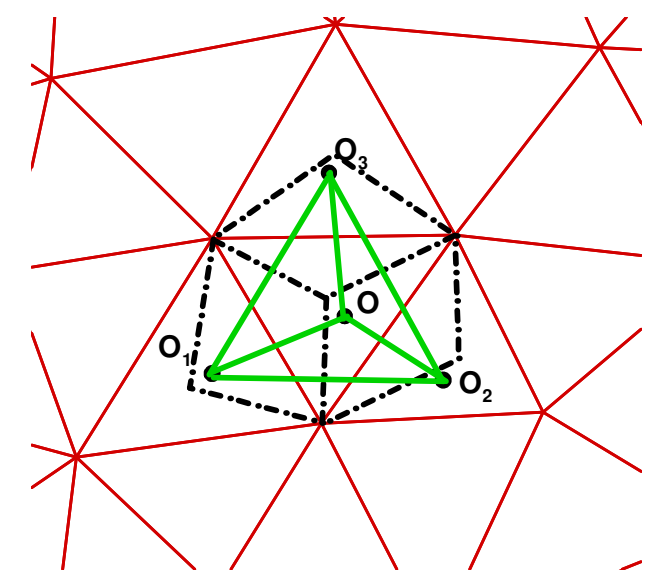

Figure 2. Triangular elements along with neighboring solution points used for derivative calculations.

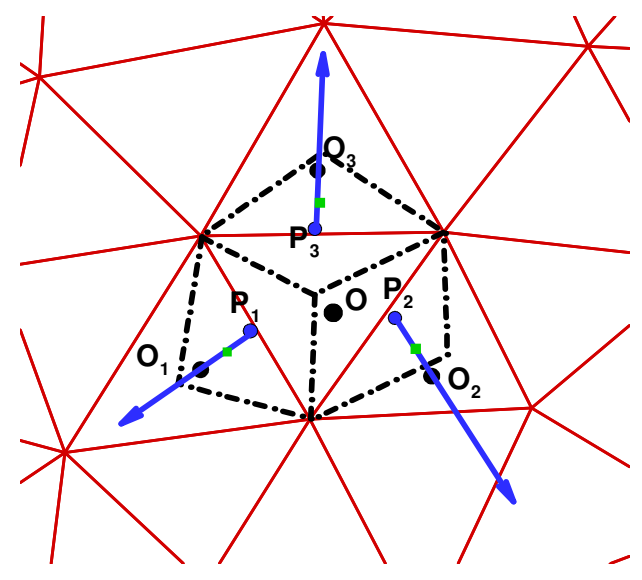

Figure 3. Triangular elements showing neighboring solution points along with zerodissipation locations used for the CNI scheme.

Despite the use of a similar weighting function, the CESE method outlined above is fundamentally different from the conventional upwind schemes due to the following properties:

1) Discretizations in space and time are treated equally. This guarantees a uniform order of temporal as well as spatial accuracy.

2) Flux conservation is enforced for all three surrounding CE's of the triangular element $F B D$ in the above analysis. When the discretized flux conservation equation (eq. (5)) is applied repeatedly for all the elements in the domain, flux conservation is guaranteed for the entire space-time domain of interest.

3) Numerical integration of the conservation laws is carried out through staggered conservation elements. The interfaces of these conservation elements do not cut through different solution elements where the first-order approximation using eq. (4) is valid. As a result, there is no need to formulate a Riemann problem and reconstruct fluxes at the interfaces, as is done for almost all finite-volume or DG methods. Aside from the difficulties in constructing a "genuine" multi-dimensional Riemann solution, any special interface treatment during flux integration produces inconsistency in numerical integration over the entire domain. 
4) Combining properties 2 and 3, the CESE method offers, arguably, the only genuine multi-dimensional scheme todate based on finite-volume integration using the strong form. As will be demonstrated later, a genuine multidimensional scheme ensures consistency and accuracy in dealing with strong flow discontinuities.

5) The CESE method offers a discretized scheme that preserves flux conservation not only in all spatial directions, but also in time. It has been shown that the space-time conservation property leads to a much more forgiving boundary treatment (e.g. ref.[22]). In many unsteady simulations, the boundary reflection is relatively minor and confined within a small region near the boundary. A direct benefit is that the proximity of the truncated boundary is not an issue for many practical applications.

\section{The Courant Number Insensitive (CNI) scheme}

Despite the effectiveness of the c-scheme, it can be shown that numerical dissipation increases as the local CFL number decreases [1]. For highly non-uniform meshes, numerical accuracy is significantly impacted by large numerical dissipation. Chang [27] introduced the Courant number insensitive scheme (CNI) scheme to deal with small CFL numbers. In the CNI scheme, the neighboring solution points $\left(O_{1}, O_{2}\right.$, and $\left.O_{3}\right)$ used for finite difference derivative evaluation are parametrically shifted to control numerical dissipation. Referring to Fig. 3, let points $P_{1}, P_{2}$, and $P_{3}$ denote the geometric centers of quadrilaterals $G B A F, G F E D$, and $G D C$, respectively. Points $N_{i}$, as defined by

$$
N_{i}=P_{i}+\sigma\left(O_{i}-P_{i}\right)
$$

are used for derivative evaluation instead of solution points $O_{i}$. The parameter $\sigma$ is used to control numerical dissipation by moving the point $N_{i}$ along the ray $P_{i} O_{i}$. It can be shown that the points $P_{i}$ correspond to "zero" dissipation when the time step is approaching zero [27]. Therefore, by using a $\sigma$ value smaller than unity, numerical dissipation can be reduced. On the other hand, a $\sigma$ value of unity is equivalent to the original c-scheme. In his investigation of Navier-Stokes solutions, Chang [22] pointed out that using a $\sigma$ value greater than unity will increase the numerical dissipation beyond what the c-scheme offers. It is crucial to use a larger $\sigma$ value in a high-aspect ratio mesh near viscous wall to maintain numerical instability. Numerical experiments performed by Chang [22] also indicate that the use of geometric centers for $P_{i}$ may not be as robust as a scheme that uses the midpoints between solution points, i.e.,

$$
P_{i}=\left(O+O_{i}\right) / 2
$$

All solutions presented in ref. [22] were carried out by using Eq. (11), not the geometric centers of surrounding conservation elements.

In the CNI scheme, evaluation of neighboring solutions are now replaced by

$$
\tilde{Q}_{0}^{i}=Q_{0}^{i}+Q_{t}^{i} \Delta t / 2+Q_{x}^{i}\left(x_{N_{i}}-x_{i}\right)+Q_{y}^{i}\left(y_{N_{i}}-y_{i}\right)
$$

where $Q_{x}^{i}$ and $Q_{y}^{i}$ are solution derivatives at the neighboring solution element and $\left(x_{N_{i}}, y_{N_{i}}\right)$ denotes the coordinates of the point $N_{i}$. Neighboring derivatives can then be evaluated by

$$
\begin{aligned}
& \left(Q_{x}^{1}, Q_{y}^{1}\right)=f_{d}\left(Q_{0}, \tilde{Q}_{0}^{1}, \tilde{Q}_{0}^{2}, O, N_{1}, N_{2}\right) \\
& \left(Q_{x}^{2}, Q_{y}^{2}\right)=f_{d}\left(Q_{0}, \tilde{Q}_{0}^{2}, \tilde{Q}_{0}^{3}, O, N_{2}, N_{3}\right) \\
& \left(Q_{x}^{3}, Q_{y}^{3}\right)=f_{d}\left(Q_{0}, \tilde{Q}_{0}^{3}, \tilde{Q}_{0}^{1}, O, N_{3}, N_{1}\right)
\end{aligned}
$$

The same weighting functions, Eqs. (8-9), can be used to compute the solution derivatives as before.

For a relatively uniform mesh, the CNI scheme can be used to control numerical dissipation when the CFL number 
is approaching zero. A $\sigma$ value of 0.01 or even smaller can be used when the CFL number is on the order of 0.01 or smaller. However, for a highly stretched mesh with a very high aspect ratio near viscous wall, a small $\sigma$ value in the large size mesh region may result in numerical instability. In practice, the $\sigma$ value may not be smaller than 0.3-0.4. An optimal distribution of $\sigma$ with respect to the local CFL number has yet to be determined. Depending upon the aspect ratio variation, typical values of $\sigma$ range from on the order of 100 to a minimum value of about 0.4 for Navier-Stokes calculations [22].

An alternative to the CNI scheme for steady-state calculations is to use local time-stepping. In these calculations, the local time step varies from element to element, but the local CFL number is kept at a constant value. While this causes some inconsistency in the temporal derivatives, the numerical solution should be the same once a steady-state is reached. Local time-stepping can speed up convergence to steady state in most applications. For a highly nonuniform mesh, a much larger value of $\sigma$ is needed only for very high aspect ratio elements and should be kept small for other elements even if local time-stepping is used. In this case, numerical experiments indicate that by varying $\sigma$ according to the mesh size and the aspect ratio (using larger values for larger aspect ratios) can also improve the solution accuracy and convergence to steady-state.

\section{The Edge-based Derivative (EBD) Approach}

The advent of the CNI scheme makes CESE computations on a stretched mesh more accurate if no local timestepping is used. However, numerical problems still exist when a high aspect ratio mesh is encountered. For a typical viscous flow calculation, when the aspect ratio near the no-slip wall is more than 100, a large $\sigma$ value on the order of 10-100 is needed to stabilize the time-marching procedure. If the aspect ratio goes above $10^{4}$ or so, the time iteration becomes unstable no matter how large the $\sigma$ is. This numerical instability is associated with the derivative calculation in the second step (eq. (7)), not the flux integration in eq. (5). The situation is better illustrated in Fig. 4 where a typical viscous mesh is shown. As can be observed, some of the neighboring solution points form an illshape triangle (e.g. $\triangle O O_{1} O_{2}$ and $\triangle O O_{3} O_{I}$ ) in conjunction with the element solution point $O$. Two major problems arise due to these ill-shape triangles. First, dependent variable derivatives (e.g. $f_{d}\left(Q_{0}, \widetilde{Q}_{0}^{1}, \widetilde{Q}_{0}^{2}, O, O_{1}, O_{2}\right)$ in eq. (7)) using these solution points may result in significant error if the three points involved are almost co-linear. This causes the derivatives computed in the second stage to have a very large values. Large derivatives in turn cause large error in the flux integration for the next time step, and the iteration eventually becomes unstable.

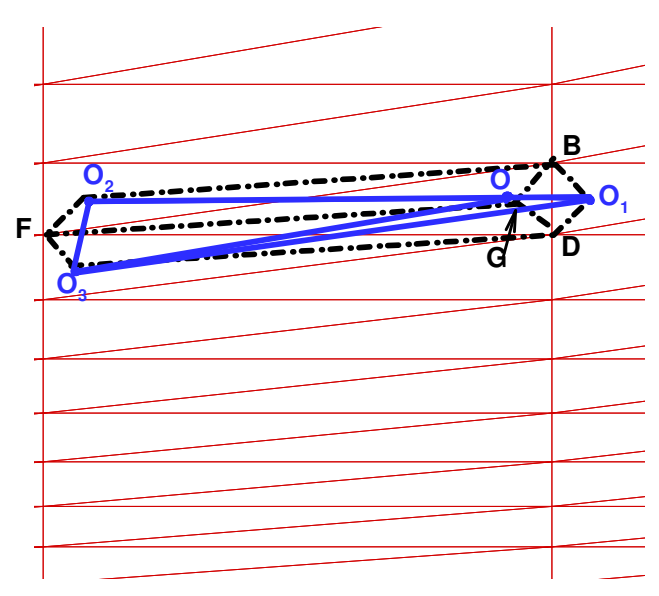

Figure 4. Conservation elements and neighboring solution points for a high aspect ratio mesh, showing skewness of the triangles used for derivative calculations.

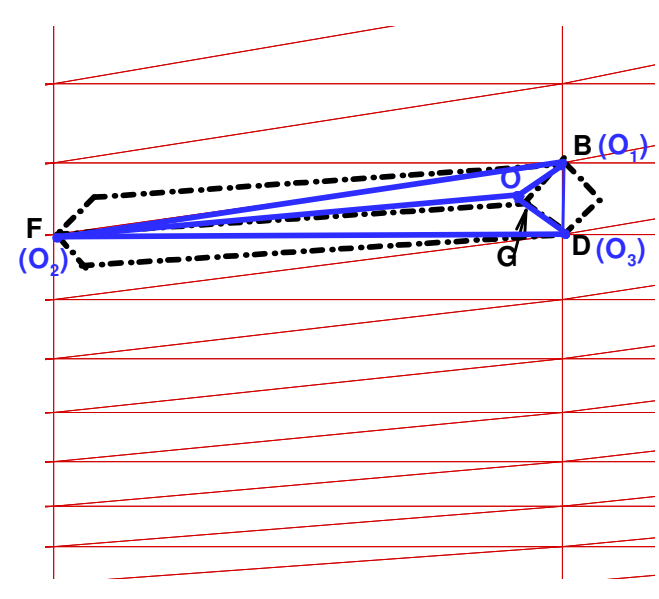

Figure 5. Conservation elements and neighboring points used for derivative calculations in the edge-based derivative approach. 
The second, and probably more important issue is related to the shape and location of the triangles used for the derivative calculations. As shown in Fig. 4, dependent variable derivatives computed at the current time level would be used to calculate fluxes on the interfaces of three quadrilateral-shaped CE's (depicted with dash lines in the figure) at the next time level. For instance, flux integration over the interface on the line segment $G B$ would need to use eq. (4) and the computed derivatives, $Q_{x}$ and $Q_{y}$. However, these derivatives are computed using three triangles formed by $\mathrm{O}_{1} \mathrm{O}_{2} \mathrm{O}_{3}$. None of these three triangles cover the line segment $G B$ entirely. A similar observation can be made for line $G D$. Only the line segment $G F$ is located mostly within the triangles used for derivative calculations. For a small aspect ratio mesh, as shown in Fig. 2, this inconsistent shape and location may not have major effect on the accuracy of derivatives. However, in a typical viscous calculation, the situation is much worse than that shown in Fig. 4. Consequently, inaccurate derivatives used for flux integration may lead to numerical problems during time marching.

A better strategy to improve the accuracy is to use a consistent shape and location for both the derivative evaluation and flux vector integration. This notion forms the basis for the modified scheme using edge-based derivatives. In this new approach (see Fig. 5), we use the vertices of the triangular element instead of the neighboring solution points for derivative calculations. In other words, points $O_{1}, O_{2}$, and $O_{3}$ now coincide with $B, F$, and $D$, respectively. Equivalently, derivative evaluation is now based on three edges of the triangular element. For the side $\overline{D B}$ (or $\overline{O_{1} O_{2}}$ ) that is located within the neighboring solution element $I$ (with solutions defined by $Q_{0}^{I}, Q_{t}^{I}, Q_{x}^{I}$, and $Q_{y}^{I}$ at a solution point $\left.\left(x_{I}, y_{I}\right)\right)$, solutions at the two vertices are given by

$$
\begin{aligned}
& \tilde{Q}_{I}^{1}=Q_{0}^{I}+Q_{t}^{I} \Delta t / 2+Q_{x}^{I}\left(x_{O_{1}}-x_{I}\right)+Q_{y}^{I}\left(y_{O_{1}}-y_{I}\right) \\
& \tilde{Q}_{I}^{2}=Q_{0}^{I}+Q_{t}^{I} \Delta t / 2+Q_{x}^{I}\left(x_{O_{2}}-x_{I}\right)+Q_{y}^{I}\left(y_{O_{2}}-y_{I}\right)
\end{aligned}
$$

The corresponding derivatives are then

$$
\left(Q_{x}^{I}, Q_{y}^{I}\right)=f_{d}\left(Q_{0}, \tilde{Q}_{I}^{1}, \tilde{Q}_{I}^{2}, O, O_{1}, O_{2}\right)
$$

Repeating the above procedure for the remaining two sides with neighboring elements $I I$ and $I I I$ gives,

$$
\begin{aligned}
& \left(Q_{x}^{I I}, Q_{y}^{I I}\right)=f_{d}\left(Q_{0}, \tilde{Q}_{I I}^{2}, \tilde{Q}_{I I}^{3}, O, O_{2}, O_{3}\right) \\
& \left(Q_{x}^{I I I}, Q_{y}^{I I I}\right)=f_{d}\left(Q_{0}, \tilde{Q}_{I I I}^{3}, \tilde{Q}_{I I I}^{1}, O, O_{3}, O_{1}\right)
\end{aligned}
$$

Finally, the derivatives in the element can be computed by applying a weighted average over the above three sets of derivatives just obtained. In contrast to the original CESE method, the current edge-based derivatives approach calculates dependent variable derivatives based on the element edges. These edge derivatives allow neighboring elements to influence the solution at the derivative level. The main advantage of this new approach is that derivatives are evaluated using a shape and location that covers all interfaces of the CE. Comparing Figs. 4 and 5, it is evident that the misalignment of the triangles for derivative evaluation and flux integration does not occur for the new approach.

In fact, the use of the side faces and element vertices in the derivative evaluation as outlined above corresponds to a zero dissipation state when the time step is approaching zero, analogous to the use of geometric centers of the CE's in the CNI scheme ( $P_{i}$ in Fig. 3). Numerical experiments also confirmed this observation. For practical Euler or Navier-Stokes calculations, numerical dissipation may be added in a fashion similar to the CNI scheme. Instead of using vertices of the triangular element, an adjustable parameter $\sigma$ is used to control the points $N_{i}$ along the rays $\overrightarrow{O O_{1}}, \overrightarrow{O O_{2}}$, and $\overrightarrow{O O_{3}}$ according to

$$
N_{i}=O+\sigma\left(O_{i}-O\right)
$$


Unlike the original CNI scheme discussed above, a $\sigma$ value of unity (using points $O_{i}$ for $N_{i}$ ) now corresponds to "zero" numerical dissipation. As shown in Fig. 6, the use of a $\sigma$ value greater than 1 effectively expands the triangles used for derivative evaluations. An equivalent CNI scheme for the EBD approach can thus be constructed by adjusting the $\sigma$ value according to the local CFL number.

\section{Validation of the EBD Courant Number Insensitive Scheme}

To test the validity of the Courant number insensitive scheme based on the proposed EBD approach, we compute a supersonic flow over a $5^{0} \mathrm{ramp}$ with a stretched mesh as shown in Fig. 7. The solution computed by using the original c-scheme with a CFL number of 1 is shown in Fig. 8 as the baseline solution. Due to the disparity in grid size, the computed shock near the outflow boundary is significantly smeared. Figure 9 shows the solution computed by using the EBD Courant number insensitive method. The shock resolution is improved near the outflow boundary. The solution can be further improved by using the local time-stepping (constant CFL) method coupled with a locally adjusted $\sigma$ distribution (see Fig. 10). These results indicate that the concept of CNI scheme still works well for the current EBD scheme. A $\sigma$ value of in the range of 1 to 10 is normally used in typical calculations using the EBD approach. A value close to 1 while maintaining numerical stability should be used to reduce numerical dissipation. It is interesting to note that an asymptotic behavior is observed for large $\sigma$ value for some test cases when the local time-stepping procedure is used for a very high aspect ratio mesh. Numerical dissipation appears to be unchanged beyond certain value of $\sigma$.

The use of eq. (19) also plays an important role in Navier-Stokes calculations. For very high aspect ratio meshes, the EBD scheme is much more robust than the original scheme due to the reasons mentioned above. For two dimensional calculations, the EBD scheme can handle an aspect ratio as large as $10^{5}-10^{6}$. By using a large $\sigma$ value on the order of 100 or larger, numerical stability can be maintained for high-aspect-ratio (on the order of $10^{5}$ ) tetrahedral meshes. Overall, the use of EBD modification can significantly improve the robustness of threedimensional Navier-Stokes calculations, especially for tetrahedral meshes. Furthermore, the enhanced stability is obtained without any loss of accuracy.

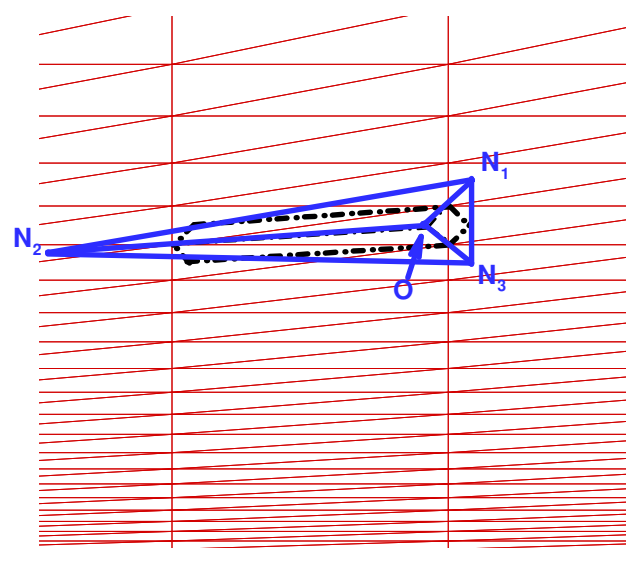

Figure 6. Construction of Courant number insensitive scheme for the edge-based derivative approach.

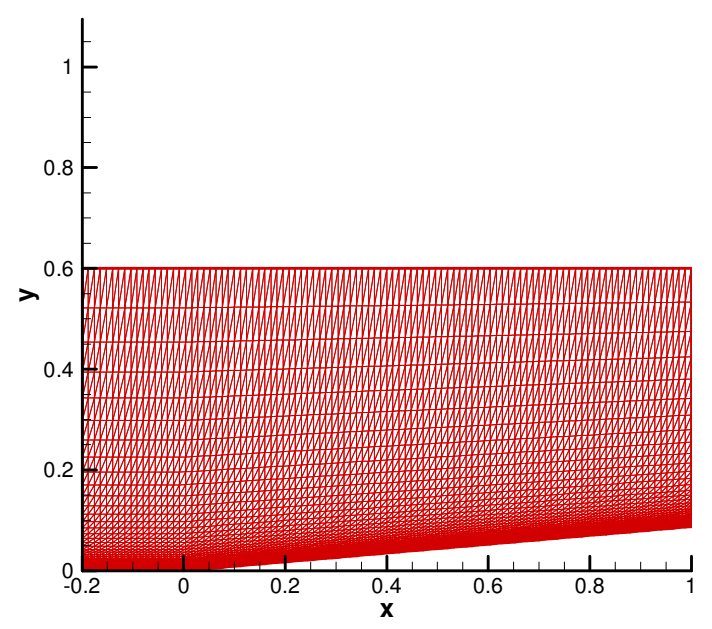

Figure 7. Unstructured triangular mesh for a Mach 3 flow over a $5^{0}$ ramp 


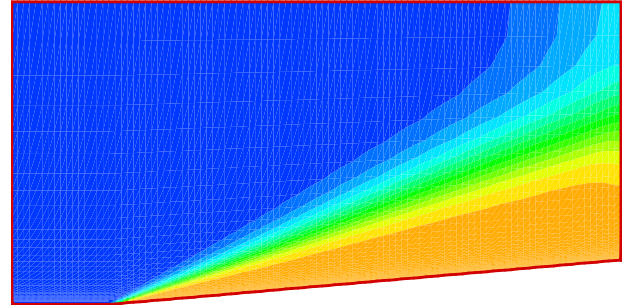

Figure 8. Density contours computed by using the original c-scheme with CFL $=1$.

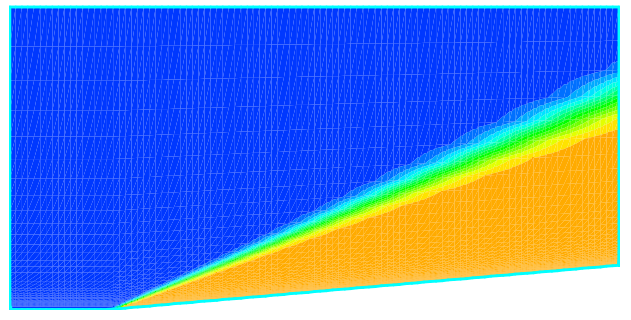

Figure 10. Density contours computed by the modified scheme with local time stepping and locally varying $\sigma$.

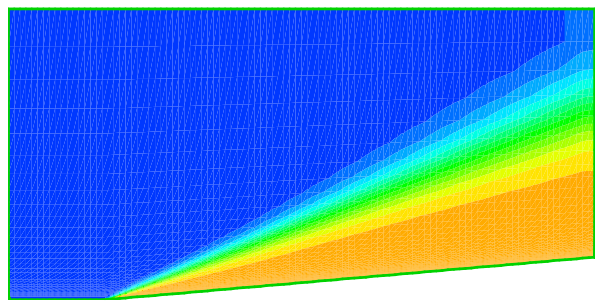

Figure 9. Density contours computed by using the modified CNI scheme with CFL $=1$.

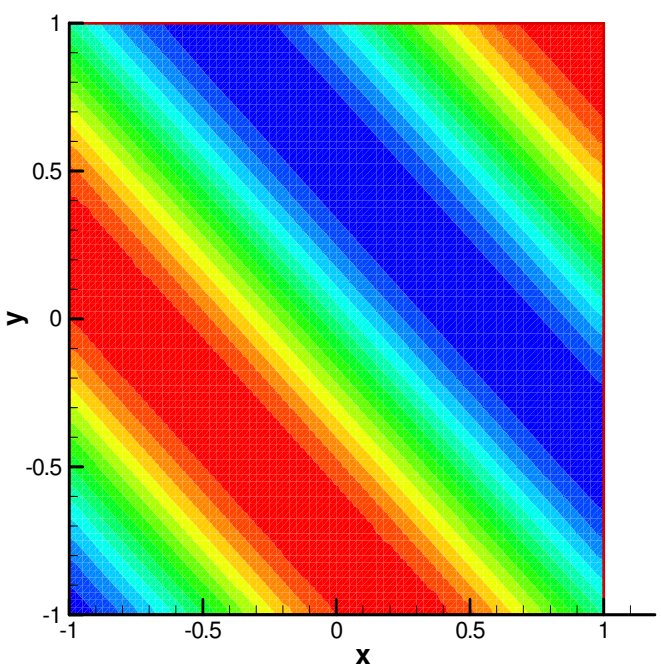

Figure 11. Oblique acoustic wave propagates diagonally through a square domain, showing density contours.

\section{Results and Discussion}

Several 2D and 3D test problems were investigated to validate the current EBD scheme. We focus on two main issues concerning the application of this modified CESE method: order of accuracy and hypersonic flow applications. The order of solution accuracy for the CESE method based on numerical results has yet to be determined in the literature. Here, we benchmark the solution accuracy for flows with and without shocks; and for steady as well as time-accurate calculations. All CESE solutions presented below were computed by using the modified EBD scheme. 


\section{A. Order of Accuracy Study}

To benchmark the order of accuracy for time-accurate calculations, we first compute an oblique acoustic wave propagating through a square domain as shown in Fig. 11. No mean flow is present in the domain. Acoustic wave inflow is specified at the left and lower boundaries and non-reflecting boundary conditions are applied at the upper and right boundaries. An unstructured mesh is generated by triangulating a corresponding uniformed structured quadrilateral mesh. For all calculations, the CFL number is fixed at 1 . Thus, the time step decreases as the grid is refined. The density contours after the wave has completely traversed the domain are shown in the figure. The computed solutions within a square box centered at the origin with a length of 1 are compared with the exact solution in order to calculate the L2 error norm shown in Fig. 12. The quantity $N$ in the figure is the number of points in both the $x$ and $y$ directions for the corresponding structured mesh. When the grid size is doubled, the error norm decreases by roughly a factor 4 or slightly better. This error norm confirms that the present scheme is indeed second order in both space and time. Although not shown here, the original c-scheme produces similar error norm convergence to the present EBD scheme.

A more challenging benchmark problem to test the solution accuracy includes a shock in the flowfield. A Mach 3 flow over a $5^{0}$ ramp is computed with a triangular mesh generated by the corresponding structured meshes $41 \times 21$, $81 \times 41,161 \times 81$, and $321 \times 161$. The resulting area-averaged L1 error norm is plotted against the number of grid points in the streamwise direction in Fig. 13. The general observation is that the error is the largest near the oblique shock and small in the smooth region. However, the area-averaged norm is dominated by a large area of smooth flow region. The overall L1-norm for large $\mathrm{N}$ is approaching second order. This example indicates that the weighted average applied in the vicinity of the shock does not have an adverse effect on the solution accuracy away from it. This is a necessary property of any shock capturing scheme.

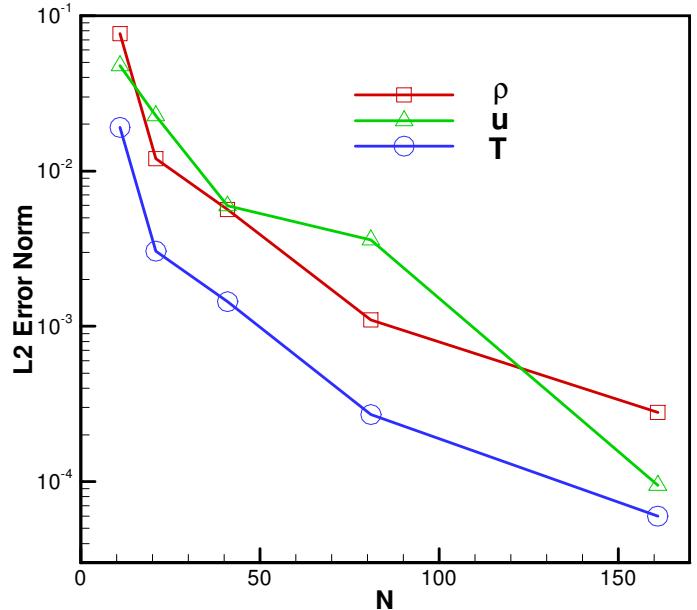

Figure 12. Convergence of error norm versus number of points in the mesh for acoustic wave propagation shown in Fig. 11.

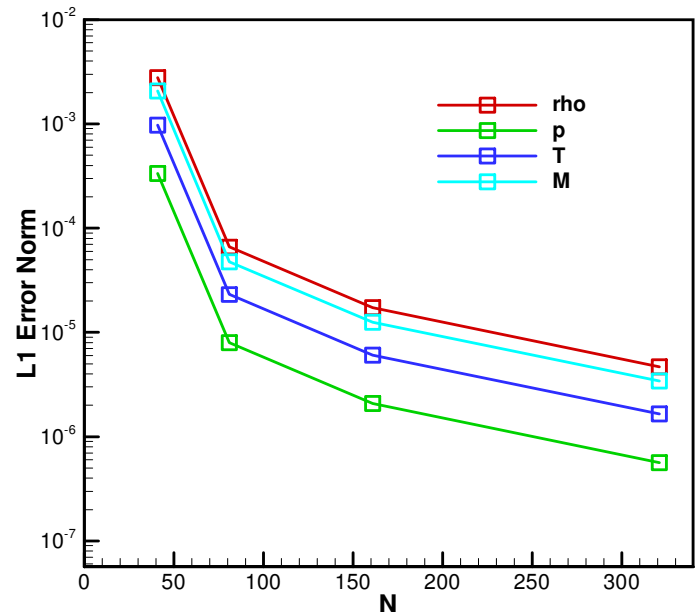

Figure 13. Convergence of error norm versus number of grid points for a Mach 3 flow over a $5^{0}$ wedge.

A more rigorous test is the accuracy of a time-accurate calculation in the presence of a shock. We investigate the one-dimensional acoustic wave and shock interaction problem normally used to assess the accuracy of the essentially non-oscillating (ENO) schemes [28]. In this case, an acoustic wave propagates through a Mach 3 moving shock wave and generates waves and shocklets behind the shock. The computed density distribution at the nondimensional time of 1.8 using 1600 points is shown in Fig. 14 along with the ENO-RF-3 solution by Shu[28] using the same grid resolution. The agreement between both sets of solutions is good. Both the shorter scale near the shock and $\mathrm{N}$-shaped shocklets further upstream are properly resolved by the current second order scheme. Figure 15 shows the computed L1 error norm (computed with the solution obtained by using 3200 points as the reference) 
versus number of points used. From the rate of change of the curve, the solution is only slightly better than first order. Apparently, the shock capturing weighted average results in reduced solution accuracy near the discontinuity. This result appears to be in line with Casper and Carpenter's [29] conclusion drawn from time-accurate wave/shock interaction inside a 1D nozzle. First-order accuracy in the presence of flow discontinuity may appear to be unsatisfactory. However, the fact that only a slightly higher than first-order convergence rate was obtained by the fifth-order weighted ENO (WENO) scheme (Pirozzoli [30]) implies that the present result is as good as could be expected for this difficult problem. By visually comparing the current solutions with those given in ref.[28], it is evident that the present solution using 800 points is comparable with the one obtained with 400 points using the ENO-RF-3 scheme, and is much better than the 800 point solution computed with the MUSCL type TVD scheme shown in the paper.

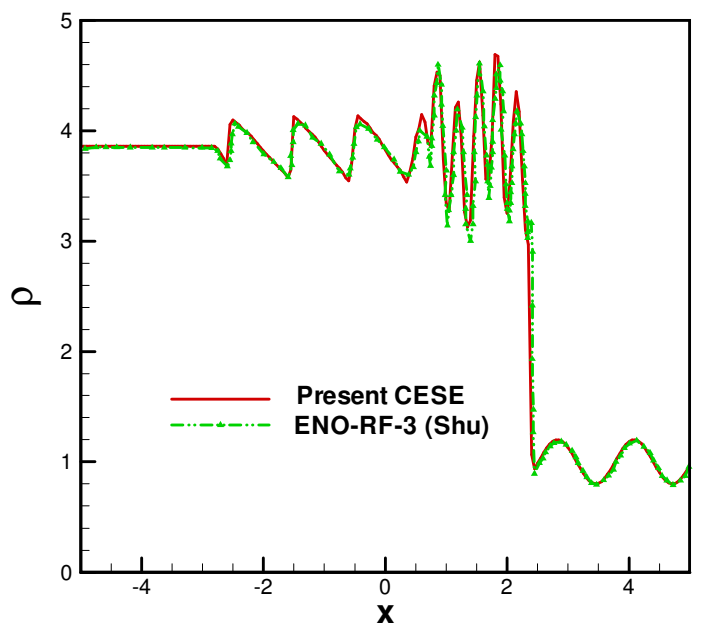

Figure 14. Density distribution at $\mathrm{t}=1.8$ for the one-dimensional acoustic wave and Mach 3 moving shock interaction.

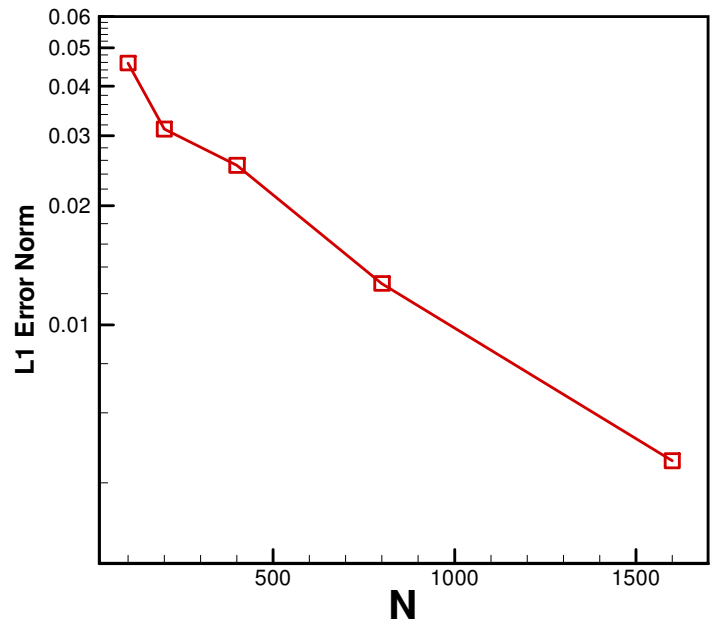

Figure 15. L1 error norm versus grid resolution for the 1D acoustic wave/shock interaction shown in Fig. 14.

Another test problem usually used to benchmark the accuracy of the ENO scheme is the 2D shock-vorticity wave interaction investigated in refs.[28, 30]. In this test case, a vorticity wave interacts with a Mach 8 moving shock wave in a rectangular domain. A shock is initially located at $x=-1$ and is moving into a region where the flow state is defined by

$$
\begin{aligned}
& \rho=1 \\
& u=-\sqrt{\gamma} \sin \theta \cos (k x \cos \theta+k y \sin \theta) \\
& v=\sqrt{\gamma} \sin \theta \cos (k x \cos \theta+k y \sin \theta) \\
& p=1
\end{aligned}
$$

The values of $k$ and $\theta$ are $2 \pi$ and $\pi / 6$, respectively. Periodic boundary conditions are applied at the top and bottom boundaries. A triangulated uniform $384 \times 256$ structured mesh is used for computations. Figure 16 shows the computed density contours at a non-dimensional time of 0.2 . The comparison with the exact solution along the $y=0$ line is depicted in Fig. 17. The wave structure is properly resolved by using the current scheme, and the agreement with the exact solution is good except for missing the sharp gradient near $x=0.55$. Comparing with solutions obtained by the WENO scheme in ref. [30] indicates that with half of their mesh size, the present solution matches roughly their W5 (fifth-order) WENO scheme. The 7-th order schemes of [30] give a slightly better solution near $x=$ 0.55 . 


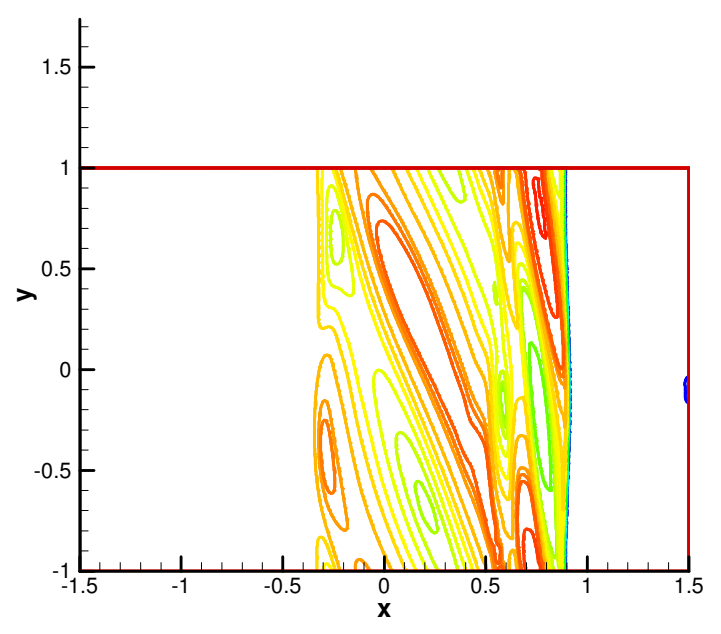

Figure 16. Computed density contours for the shock-vorticity wave interaction problem at $\mathrm{t}=$ 0.2 , the shock is initially located at $x=-1$.

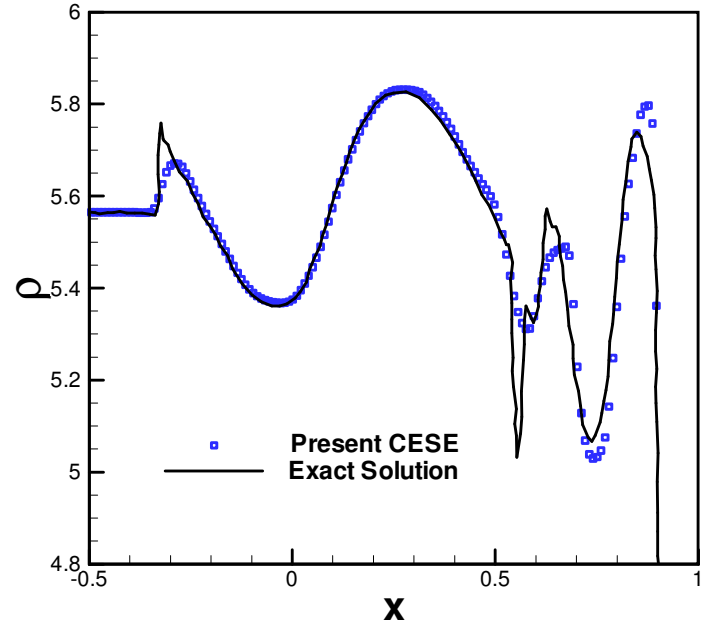

Figure 17. Comparison of density distribution along $y=0$ with exact solution for the shockvorticity wave interaction problem shown in Fig. 16.

\section{B. Hypersonic Blunt Body Computations}

In the previous section, we have demonstrated the solution accuracy of the present scheme for several time-accurate and steady-state benchmark problems with flow discontinuities. This section is focused on hypersonic, blunt-body computations. Accurately computing hypersonic blunt body flows and maintaining numerical stability is one of the outstanding issues related to hypersonic flow simulations using unstructured meshes. Despite decades of success in computing bow shocks using upwind schemes with structured meshes, there are still reports of the famous carbuncle phenomenon in which numerical instability associated with the stagnation region behind the shock causes the shock to become unstable [16-17, 20-21]. For the configurations where stable solutions can be obtained, the carbuncle phenomenon may still be observed if more grid points are used between the shock and body in the calculation [31]. For the finite-volume or DG based unstructured methods, this numerical instability appears to be a more serious issue (e.g. refs.[16-17]). Due to the scope limitation, we only focus on two issues related to blunt body computations: 1) the order property of the present scheme in calculating bow shocks 2) assessment of the solution quality for hypersonic flow over a cylinder with strong surface heat transfer.

A Mach 6 flow over a circular cylinder is chosen to study the order of accuracy of the present scheme. The baseline solution used to benchmark the order of accuracy is obtained by a shock-fitting code (Salas and Atkins [32]). In such shock-fitting calculations, a discretized domain between the shock and the body is used in solving the Euler equations. The main advantage of this approach is that any issues related to numerical instability and the accuracy constraint of the shock capturing scheme do not play any role. If sufficient points are used, a "clean" solution can thus be regarded as an exact solution. Structured meshes with dimensions 31x41 (31 along the cylinder surface and 41 along the wall-normal direction), 61x81, 121x161, and $241 \times 321$ are triangulated for the present CESE calculations. Figure 18 shows the $31 \times 41$ mesh near the symmetry line. In these Euler calculations, the slip wall condition is used on the cylinder surface. Free-stream and symmetry conditions are applied at the inflow and center line, respectively. Figures 19 and 20 compare the computed Mach numbers and pressure contours using the finest grid (241x321) with the shock-fitting solution using a $192 \times 320$ mesh. Excellent agreement between these two methods is evident.

The shock-fitting solution provides an excellent baseline for the validation of several variations of the CESE method. Numerical results indicate that using the in-centers instead of the geometric centers of the triangular element to construct the surrounding CE's (points $G, A, C$, and $E$ in Fig. 1) is apparently more accurate. A 
triangular mesh is also more accurate than a comparable quadrilateral mesh with the same number of elements for this Mach 6 bow shock problem. Figure 21 depicts the L2 error norm for various mesh sizes. These error norms were calculated at the shock-fitting calculation grid points on the cylinder surface and along the symmetry line. The CESE solutions at these discrete points were calculated by using eq. (4). As suggested in ref. [32], using an integral quantity for the error norm calculation is to be avoided to ensure appropriate measure of order properties. The rate of change of both curves shown in Fig. 21 indicates only about first-order accuracy. An area-averaged error norm as computed for the supersonic flow over a wedge (Fig. 13) is not available for this case. The reason for the lower than second order accuracy is unknown. However, the non-orthogonal mesh near the stagnation region (see Fig. 18) may affect the solution accuracy of the present scheme.

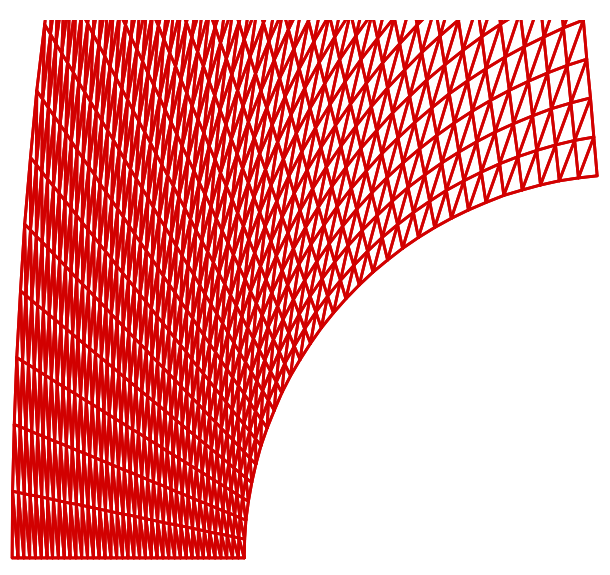

Figure 18. Triangular mesh used for the Mach 6 bow shock calculation, showing the coarsest $31 \times 41$ mesh.

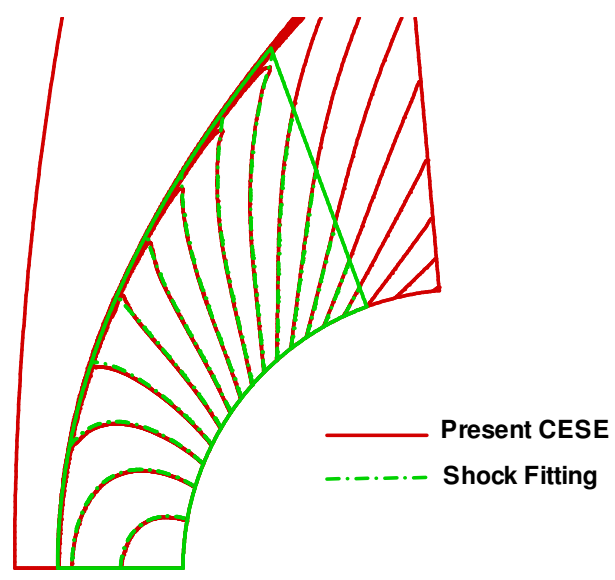

Figure 20. Comparison of the pressure contours computed by the shock-fitting code and the present method for the Mach 6 flow over a cylinder.

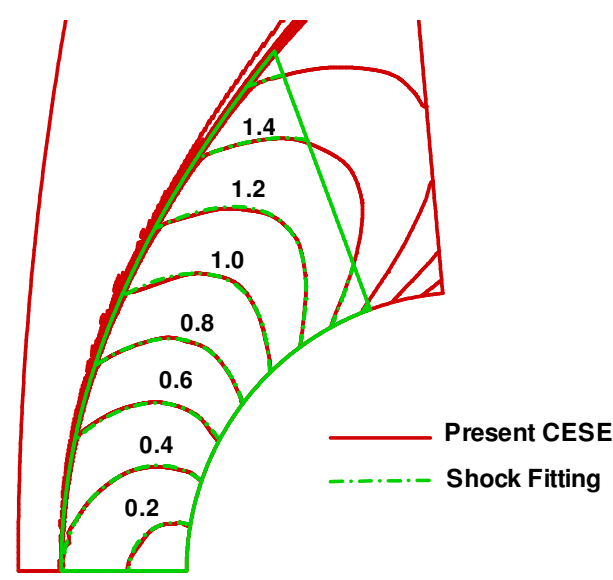

Figure 19. Comparison of the Mach number contours computed by the shock-fitting code and the present method for the Mach 6 flow over a cylinder. 
As pointed out in [16], aerothermodyamic simulation of a blunt body using unstructured grids still poses numerical issues associated with the carbuncle phenomenon and the ineffectiveness of the entropy fixes. To accurately predict surface heat transfer, a highly refined mesh near the body surface must be used. The configuration used by the LAURA benchmark [16] is a Mach 17 flow over a 2D or 3D cylindrical surface. Figure 22(a) shows the triangulated $61 \times 65$ mesh used for simulation. The highest aspect ratio near the wall is about 1400 , and, as a result, a $\sigma$ value on the order of 100 is needed to achieve a converged solution. Figure 22(b) shows the computed temperature contours around the body after the L2 norm has dropped 5 orders of magnitude. As shown, the mesh used is intended to properly resolve both the bow shock and thermal boundary layer. Figure 23 shows a comparison of cylinder surface heating and non-dimensional pressure between the present results and that from the LAURA code [16]. The overall agreement is marginal, especially with the surface heating being under-predicted. However, the present solution is by no means a grid-converged solution. Another run with a higher grid stretching near the wall while keeping the same 65 points in the wall-normal direction appears to over-predict the surface heating rate. This indicates that the solution shown here is not grid-converged. Nevertheless, the smooth surface heating and pressure distributions in Fig. 23 confirm that the present method does not have numerical stability problems in handling hypersonic flows over a $2 \mathrm{D}$ blunt body flows with strong surface heat transfer.

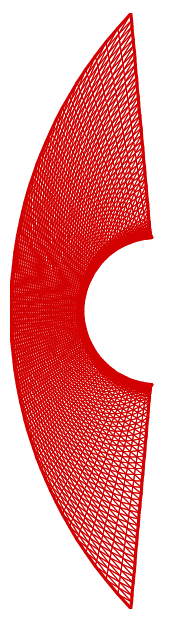

(a)

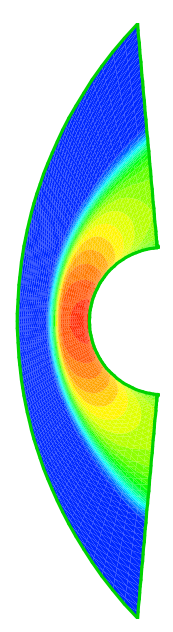

(b)

Figure 22. Triangular mesh and computed temperature contours for a Mach 17 flow over a cylinder: (a) mesh (b) temperature contours.

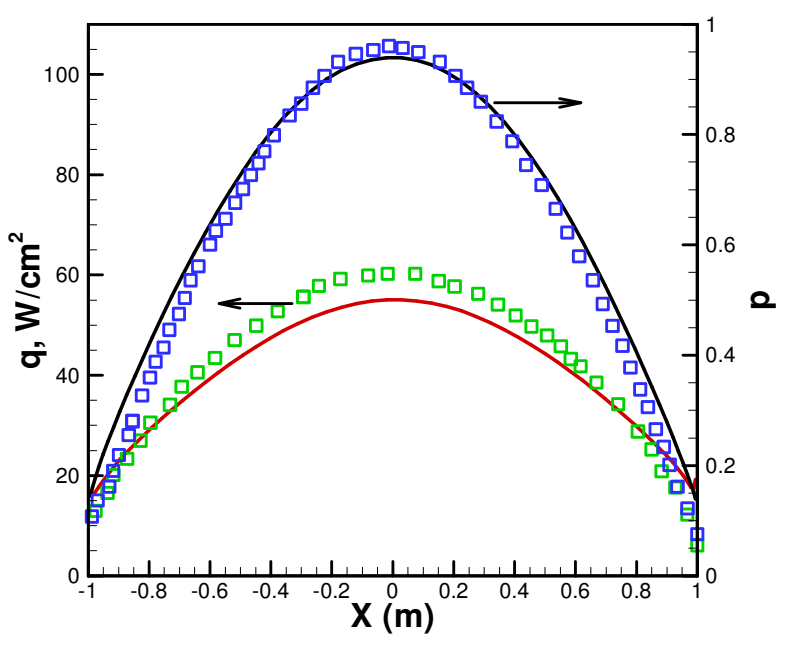

Figure 23. Comparison of surface heating and non-dimensional surface pressure with LAURA results [16]: symbols for LAURA and lines for CESE.

To demonstrate that the scheme does not suffer from stability problems in three dimensions, the above 2D mesh is stacked up 10 times along the spanwise direction to form a tetrahedral 3D mesh as shown in Fig. 24(a). Periodic boundary conditions are imposed at the spanwise boundary. The computed temperature contours are shown in Fig. 24(b). Although not visible on the scale of the figure, a weak spanwise gradient does exist in the solution. The largest spanwise gradient is taking place between the first two planes near the spanwise boundaries. Using a reflecting (slip wall) boundary condition does not seem to help in eliminating this weak gradient. The directional bias of a tetrahedral mesh may have contributed to this spanwise gradient, but more studies are necessary to confirm this. Figure 25 shows a comparison of the computed 3D surface heat transfer and non-dimensional pressure with those obtained from the 2D simulation shown in Fig. 23. Clearly, the weak spanwise gradient does affect the overall solution. In light of the differences in the discretized volumes between the $2 \mathrm{D}$ and $3 \mathrm{D}$ simulations (unless a prismatic mesh is used, the 3D tetrahedral mesh does not replicate the 2D mesh), we do expect some sort of discrepancies. Unlike the surface pressure, the heat flux computed at various spanwise planes appears to be varying. The middle planes away from the boundaries seem to agree well and peak around $40 \mathrm{~W} / \mathrm{cm}^{2}$. This value is even smaller than that from the $2 \mathrm{D}$ results. To obtain a grid converged solution is beyond the scope of this paper. 
However, the present 3D simulation using a tetrahedral mesh does not exhibit any numerical issues concerning numerical instability nor errant behavior in the surface heating near the stagnation region. As mentioned in the previous section, the CESE method does not have any intrinsic flux splitting issues near the stagnation region. The weighted average procedure for the dependent variable gradients appears to be free from the glitches of conventional upwind schemes. As such, a genuine multi-dimensional formulation coupled with a unified weighted averaging for dependent variable gradients in the present CESE method ensures that no improper entropy generation near the bow shock is allowed to contaminate the surface heating and pressure.

Although not shown here, numerical experiments with a realistic triangular or tetrahedral mesh do not exhibit any numerical difficulties either. A mesh that is misaligned with the bow shock also can be handled very well. As discussed above, the issues associated with accurate surface heating prediction need to be sorted out. Nevertheless, the present method does offer a more robust and viable approach to compute hypersonic blunt body configurations.

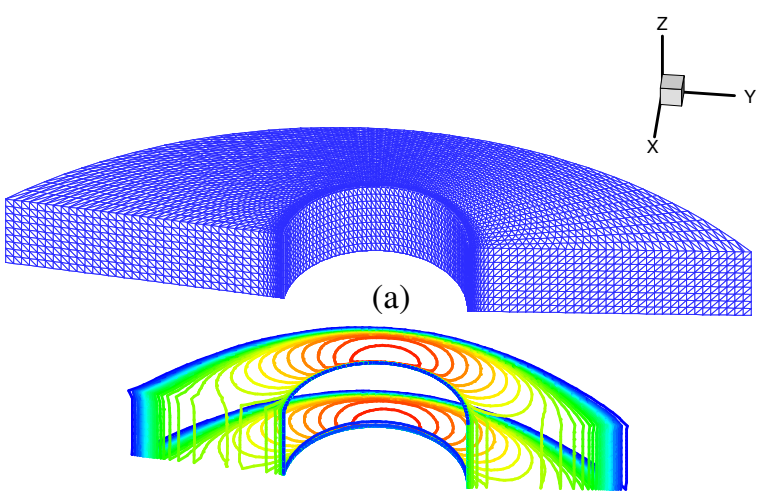

(b)

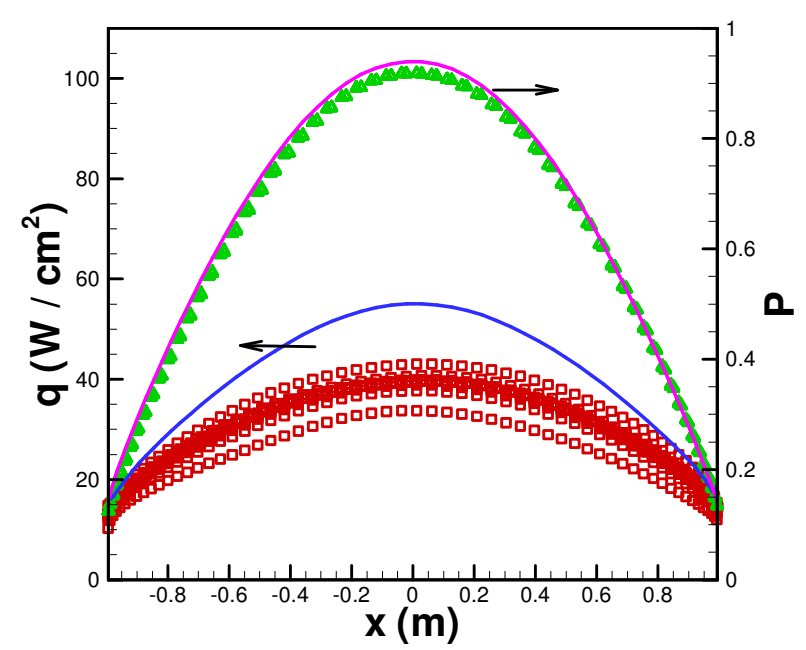

Figure 25. Comparison of computed surface heating and non-dimensional surface pressure using a 2D triangular or a 3D tetrahedral mesh.
Figure 24. Tetrahedral mesh and computed temperature contours for a Mach 17 flow over a cylinder: (a) mesh (b) contours.

\section{3D Navier-Stokes Simulations}

With the modified scheme, the CESE method now can handle very high aspect ratio viscous meshes without numerical instability. For a very high aspect ratio mesh beyond $10^{5}$, a large $\sigma$ value on the order 100-1000 must be used. This implies large numerical dissipation. Solution accuracy with such a large $\sigma$ value has not yet been assessed. However, many applications with an aspect ratio below $10^{5}$ can be computed with properly controlled numerical dissipation. The modified scheme has been fully implemented in the $e z 4 d$ code [22]. Pending validation, both Spalart-Allmaras and k-omega turbulence models are also available in this code.

Numerical tests have been performed with the modified CESE method for several 3D Navier-Stokes calculations using tetrahedral and hexahedral meshes. Figures 26(a) and 26(b) show the tetrahedral mesh and computed pressure contours for a test case of transonic flow (with a free-stream Mach number of 0.85 and $-6^{0}$ angle of attack) over a wing body configuration that has been used as a benchmark problem for the CFD Drag Prediction Workshop [33]. The results were obtained with laminar calculations. Turbulent calculations along with comparison with other drag prediction workshop solutions will be performed in the future. 

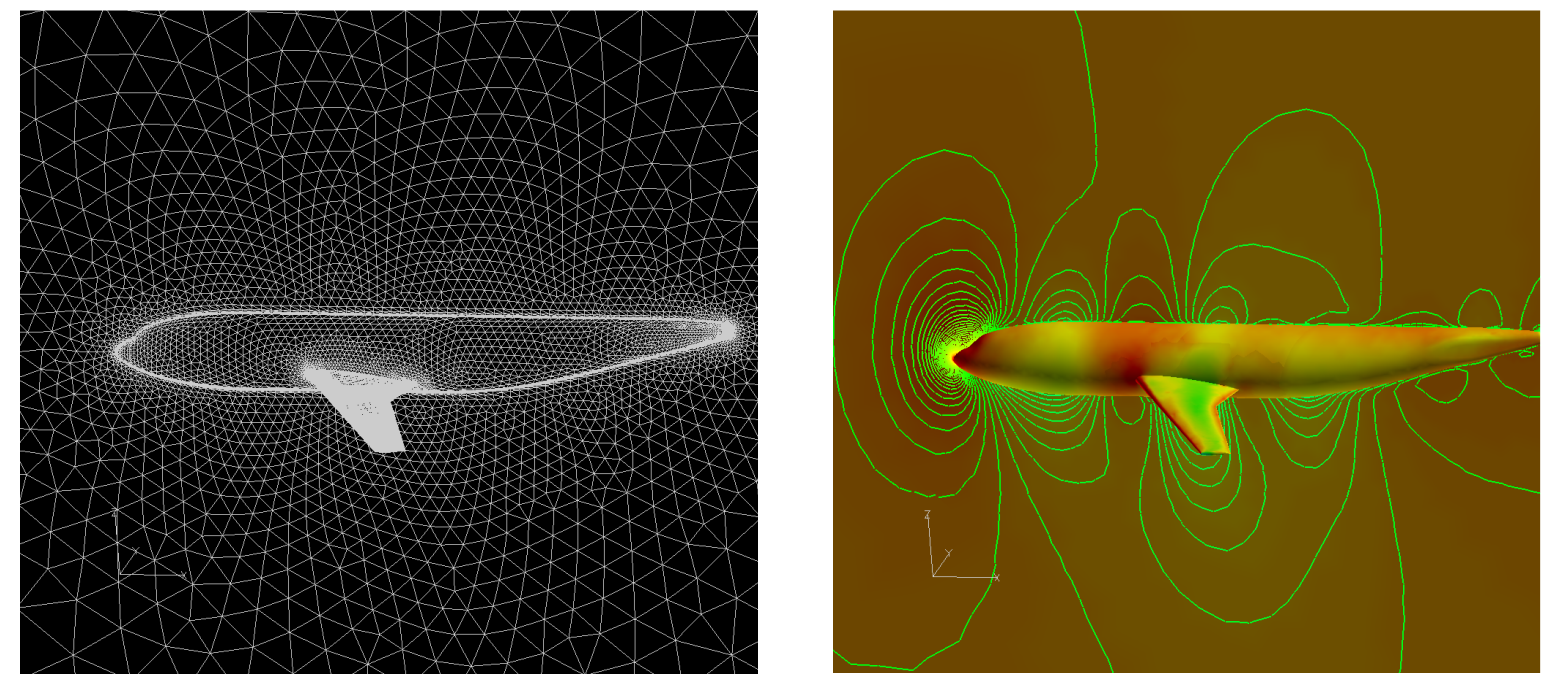

Figure 26. Tetrahedral mesh and computed pressure contours on a three-dimensional wing body configuration using the modified CESE method and the $e z 4 d$ code.

\section{Conclusion}

A new procedure to calculate the dependent variable gradients for the CESE method has been proposed. Instead of using the neighboring solution points, the modified approach uses the neighboring solution elements and the vertices of the element itself to evaluate derivatives influenced by each neighbor. The weighted average of all computed neighboring derivatives is then imposed as the resulting dependent variable gradient within each element. The shape and location used for the derivative calculations in this modified approach are similar to those used for the flux integration. As a result, dependent variable gradients may be more accurately computed for high-aspect-ratio meshes, and the time advancement procedure becomes much more robust. It has been shown that a comparable Courant number insensitive scheme and numerical dissipation control may be established by expanding the element size. A larger but similar element brings about more numerical dissipation to the solution. Numerical dissipation can be controlled by using a single parameter that determines the size of the element used for derivative calculations.

Solution accuracy of this new approach has been validated by investigating the error norm convergence for several benchmark problems: oblique acoustic wave propagation in a square domain, Mach 3 flow over a wedge, 1D shockacoustic wave interaction, and 2D shock-vorticity wave interaction problems. The results indicate that for steady state and shock-free time-accurate problems the modified scheme is second-order accurate. For a time-accurate wave and shock interaction problem, the scheme is only first order accurate. This reduced order behavior for waveshock interaction is comparable with that observed for a higher order ENO or WENO scheme.

Hypersonic flow over a cylinder is used to assess capabilities of the proposed method in handling bow shock flows robustly and accurately. Results for a Mach 6 flow over a cylinder are shown to compare very well with an "exact" solution obtained by a shock-fitting scheme. The solution accuracy along the symmetry line and the cylinder surface has been shown to be only of first order accuracy despite the steady-state nature of this test problem. Computations of a Mach 17 flow over a cylinder have been performed to assess the capability in surface heating prediction. For a $61 \times 65$ mesh, the present results are close to those obtained by the structured LAURA code[16]. Both 2D triangular and 3D tetrahedral results show consistent shock profiles and smooth surface heating distributions. Neither the carbuncle phenomenon nor abnormal surface heating distributions due to numerical issues regarding shock capturing and entropy fixes are observed. Implementation of this modified scheme in a 3D NavierStokes code shows that the present numerical framework provides a more robust method for general hypersonic flow simulations. By and large, the established solution accuracy benchmarks for acoustic wave propagation, shock and wave interaction, and blunt body flows demonstrates that the present CESE method offers a robust and accurate numerical framework for hypersonic computations using unstructured grids. 


\section{Acknowledgments}

The author is very grateful to many fruitful discussions with Dr. Sin-Chung Chang of NASA Glenn Research Center. The author would also like to thank Dr. Fei Li of National Institute of Aerospace for his assistance in grid generation and the oblique acoustic wave calculations.

\section{References}

1. Chang, S.-C., "The Method of Space-Time Conservation Element and Solution Element-A New Approach for Solving the Navier-Stokes can Euler Equations," J. Comput. Physics, Vol. 119, pp.295-324, 1995.

2. Chang, S. C., Wang, X. Y., and Chow, C. Y., "The Space-Time Conservation Element and Solution Element Method: A New High-Resolution and Genuinely Multidimensional Paradigm for Solving Conservation Laws," J. of Computational Physics 156, 89-136 (1999).

3. Loh, C. Y., Hultgren, L. S., Chang, S.C., Jorgenson P.C.E., "Noise Computation of a Shock-Containing Supersonic Axisymmetric Jet by the CE/SE Method," AIAA Paper 2000-0475, presented at the 38th AIAA Aerospace Sciences Meeting, January 10-13, 2000, Reno, NV.

4. Im, Kyoung-Su, Yu, S.-T. John, Kim, Chang-Kee, Chang, Sin-Chung, and Jorgenson Philip C.E., "Application of the CESE Method to Detonation with Realistic Finite-Rate Chemistry," AIAA Paper 20021020.

5. Zhang, Moujin, Yu, S.-T. John, Lin, S.-C. Henry, Chang, S.-C., and Blankson, Isaiah, "Solving the MHD Equations by the Space-Time Conservation Element and Solution Element Method," to appear Journal of Computational Physics, Oct. 2005.

6. Cai, Minghao, Yu, S.-T. John, and Zhang, Moujin, "Theoretical and Numerical Solutions of Linear and Nonlinear Elastic Waves in a Thin Rod," AIAA 2006-4778, 42nd AIAA/ASME/SAE/ASEE Joint Propulsion Conference and Exhibit, Sacramento, California, July 9-12, 2006

7. Ayasoufi, A., Keith, T. G., "Numerical Simulation of Melting and Freezing Processes with Density Change by the Space-Time CE/SE Method," 5th International Bi-Annual ASME/JSME Symp. on Comp. Tech. for Fluid/Thermal/Chemical/Stressed Sys. with Industrial Appl., July 25, 2004,

8. Ostman, M., "Melting Pre-study of Models and Mapping: Physical modeling of Scrap Melting," MSc Thesis, 2006, Department of Applied Physics and Mechanical Engineering, Lulea University of Technology, Lulea, Sweden.

9. Anderson, W. K. and Bonhaus, D. L., "An Implicit Upwind Algorithm for Computing Turbulent Flows on Unstructured Grid," Computers and Fluids, Vol. 23, No. 1, pp. 1-21, 1994.

10. Barth, T. J., "Numerical Aspects of Computing High-Reynolds Number Flows on Unstructured Meshes," AIAA Paper 91-0721, 1991.

11. http://fun3d.larc.nasa.gov/

12. Yen, L., Vinokur, M., and Wang, Z. J., "Multi-Dimensional Spectral Difference Method for Unstructured Grids," AIAA Paper 2005-320, 2005.

13. Cockburn, B. and Shu, C.-W., "TVB Runge-Kutta Local Projection Discontinuous Galerkin Finite Element Method for Conservation Laws II: General Framework," Mathematics of Computation, Vol. 52, No. 186, pp. 411-435, 1989.

14. Cockburn, B., Lin, S. Y., and Shu, C.-W., "TVB Runge-Kutta Local Projection Discontinuous Galerkin Finite Element Method for Conservation Laws III: One Dimensional Systems," J. Comput. Physics, Vol. 84, No. 1, pp. 90-113, 1989.

15. Atkins, H. L. and Shu, C.-W., "Quadrature-Free Implementation of Discontinuous Galerkin Method for Hyperbolic Equations," AIAA J., Vol. 36, No. 5, pp. 775-782, 1998.

16. Gnoffo, P. A. and White, J. A., "Computational Aerothermodynamics Simulation Issues on Unstructured Grids," AIAA Paper 2004-2371, 2004.

17. Darmofal, D, "Towards a High-Order Discontinuous Galerkin Method for Aerospace Applications," NASA Langley presentation, Feb. 2006.

18. White, Jeffery A. private communication

19. Wolf, W. A., "Supersonic and Hypersonic Flow Computations using High-order Non oscillatory Scheme," AIAA Paper 2006-3169, 2006. 
20. Robinet, J.-Ch., Gressier, J., Casalis, G. and Moschetta, J.-M., "Shock Wave Instability and Carbuncle Phenomenon: Same Intrinsic Origin?” J. Fluid. Mech. Vol. 417, pp. 237-263, 2000.

21. Chauvat, Y., Moschetta, J.-M., and Gressier, J., "Shock Wave Numerical Structure and the Carbuncle Phenomenon," Int. J. Numeri. Meth. Fluids Vol. 47, No. 8-9 pp. 903-909, 2004.

22. Chang, C.-L., "Time-Accurate, Unstructured-Mesh Navier-Stokes Computations with the Space-Time CESE Method," AIAA Paper 2006-4780, presented at the 42th AIAA/ASME/SAE/ASEE Joint Propulsion Conference and Exhibit, July 10-12, 2006, Sacramento, CA.

23. Wang, X.-Y. and Chang, S.-C., "A 2D Non-Splitting Unstructured Triangular Mesh Euler Solver Based on the Space-Time Conservation Element and Solution Element Method," J. Computational Fluid Dynamics, Vol. 8, No. 2, pp. 309-325, 1999.

24. Wang, X.-Y. and Chang, S.-C., "A 3D Non-Splitting Structured/Unstructured Euler Solver Based on the Space-Time Conservation Element and Solution Element Method," AIAA Paper 99-3278, 1999.

25. Hirsch, C., "Numerical Computation of Internal and External Flows," John Wiley \& Sons, 1991.

26. Chang, S.-C., "On Space-Time Inversion Invariance and Its Relation to Non-Dissipativity of a CESE Core Scheme," AIAA Paper 2006-4779, 2006.

27. Chang, S.-C. and Wang, X.-Y., "Multi-Dimensional Courant Number Insensitive CE/SE Euler Solvers for Applications Involving Highly Non-uniform Meshes," AIAA Paper 2003-5285, 2003.

28. Shu, C.-W. and Osher, S., "Efficient Implementation of Essentially Non-oscillatory Shock-Capturing Schemes, II," J. Comput. Physics, Vol. 83, pp. 32-78, 1989.

29. Casper, J. and Carpenter, M. H., "Computational Considerations for the Simulation of Shock-induced Sound," SIAM J. Sci. Comput., Vol. 19, No. 3, pp.813-828, 1998.

30. Priozzoli, S., "Conservative Hybrid Compact-WENO Schemes for Shock-Turbulence Interaction," J. Comput. Physics, Vol. 178, pp. 81-117, 2002.

31. Li, F., private communication.

32. Salas, M. D. and Atkins, H. L., "On Problems Associated with Grid Convergence of Functionals," submitted to Computers \& Fluids.

33. http://aaac.larc.nasa.gov/tsab/cfdlarc/aiaa-dpw/index.html

34. Chang, S.-C., private communication. 